\title{
Preparation of Topical Nano Gel Loaded With Hesperidin Emulsomes: In vitro and in vivo Studies
}

\author{
Yeramanchi Sarah Sujitha*, Yallamali Indira Muzib \\ Institute of Pharmaceutical Technology, Sri Padmavathi Mahila Visvavidyalayam, (Women's University), Tirupati, Andhra Pradesh, INDIA.
}

\begin{abstract}
Objectives: The objective of the present study was to increase the solubility of poorly soluble drug hesperidin and to increase the efficacy of the drug by loading hesperidin in emulsomal topical gel. Methods: Hesperidin was loaded into emulsomes by using ethanol injection method. The optimized formulation of emulsomes was evaluated by SEM, FT-IR, DSC, P-XRD, in vitro drug release, ex-vivo skin permeation studies, in vivo pharmacokinetic and pharmacodynamic studies. Results: The SEM study shows the emulsomes were tiny and spherical in structure. The particle size and zeta potential of the optimized formulation was found to be $50 \mathrm{~nm}$ and $-1.8 \mathrm{mV}$ and in vitro drug release was found to be $98.6 \%$ for $6 \mathrm{hrs}$. The optimized emulgel was prepared by using different gelling agents Carbopol 934 and HPMC. The prepared gels were found to be homogenous and skin permeation studies was found to be $98.9 \%$ for $4 \mathrm{hr}$, with skin permeation efficacy bearing flux value $12.3 \mu \mathrm{g} / \mathrm{cm}^{2} / \mathrm{h}$ when compared to the pure
\end{abstract}

hesperidin gel. The in vivo pharmacokinetic and pharmacodynamic studies were found to be more efficient for the hesperidin loaded emulsomal gel when compared to the marketed formulation. Conclusion: Hesperidin emulsomal gel has shown a significant increase $(p<0.05)$ in activity when compared to the marketed formulation.

Key words: Hesperidin, Emulsomes, Topical gel, In vitro drug release, Pharmacokinetic and pharmacodynamic studies.

\section{Correspondence}

Y. Sarah Sujitha

Institute of Pharmaceutical Technology, Sri Padmavathi Mahila Visvavidyalayam (Women's University), Tirupati-517502, Andhra Pradesh, INDIA.

Phone no: +919494462070

Email: suji.sarah@gmail.com

DOI: 10.5330/ijpi.2020.4.87

\section{INTRODUCTION}

Hesperidin is a flavanone glycoside consisting of the flavone hesperetin bound to the disaccharide rutinose which is highly detected in very young tissues of the fruit. It was first discovered by leberton that hesperidin is natural flavones present in citrus fruits. Hesperidin is an inexpensive byproduct of citrus family and it occurs in all plants including nuts, seeds, vegetables, flowers and barks. ${ }^{1,2}$ Hesperidin belonging to family Fabacea, Beulaceae and Lamiaceae. ${ }^{3,4}$ The sugar group makes hesperitin more water-soluble than hesperidin. Hesperidin has been reported to influence a wide variety of biological functions. For example, hesperidin induces apoptosis and suppresses proliferation in human cancer cells, inhibition of tumour development in various tissues including the skin. Many of these beneficial effects of hesperidin can be attributed to its antioxidant activity. Hesperidin enhances microcirculation, possess antioxidant effect, improve venous tone, anti-inflammatory, analgesic, blood lipid lowering, assist healing of venous ulcers and also used for the treatment of haemorrhoids, chronic venous insufficiency and exhibit pronounced anticancer activities. ${ }^{5-7}$ Edema is a complex biological response produced by the body tissues, which is produced during any harmful stimuli and irritants, injuries. Inflammation is characterized by pain, swelling, redness. ${ }^{8,9}$ Chronic inflammation causes the heart diseases, rheumatoid arthritis, hay fever and it is also a common manifestitation in some infectious diseases like leprosy, tubercolosis, asthma, inflammatory bowel disease and many auto immune diseases. However NSAIDS are used to treat the inflammation but the usage is limited due to the undiseable able side effects on gastric, renal, cardiovascular system. ${ }^{10,11}$

Emulsomes are smaller in nano size when compared to other vesicular drug delivery systems like ethosomes, phytosomes and they show enhanced bioavalibilty due to their reduced size. Emulsomes are a new generation of colloidal carrier composed of solid lipid surrounded by phospholipid bilayers which represent a novel lipoidal vehicle of particulate structure with improved loading capacity for drugs and biologics containing components that have been safely used to deliver medications to people. ${ }^{12,13} \mathrm{~A}$ key feature of emulsomes is that this internal core is composed of a lipid in a solid or liquid crystalline phase, rather than an oil-in-fluid phase, at $25^{\circ} \mathrm{C}$ i.e., high hydrophobic drug loading in the internal solid lipid core and the ability to encapsulate watersoluble medicaments in the aqueous compartments of surrounding phospholipid layers. ${ }^{14}$ Emulsomes may be used for parenteral, oral, occular, rectal, vaginal, intranasal, or topical delivery of either fatsoluble or water-soluble substances. ${ }^{15}$ The particle size distribution of emulsomes, based on differential weight per cent, is in the range of 10$250 \mathrm{~nm}$, making them suitable for intravenous administration. They can encapsulate water-soluble pharmaceuticals in the aqueous compartments of the outer phospholipid layers, while their cores can be loaded with high levels of hydrophobic drugs. Emulsomes thereby increase the solubility and improve the bioavailability of lipophilic drugs and their organization facilitates sustained or controlled drug release. Therefore, emulsomes may serve as efficient drug delivery systems because they exhibit good biocompatibility, biodegradability, stability, high entrapment efficiency and sustained drug release. Emulsomes have main advantages of high drug loading capacity, improved pharmacological activity, site specificity and reduced toxicity. To assess the performance of emulsomes in improving the bioavailability and prolonging systemic exposure of hesperidin, we prepared emulsomes containing hesperidin 
and evaluated their pharmacokinetic properties and in vivo drug delivery performance.

\section{MATERIALS AND METHODS}

\section{Materials}

Hesperidin was obtained as a gift sample from Virdev Pharma Pvt. Ltd., Gujarat. Stearic acid, soya lecithin and cholesterol were purchased from SD. Fine-chem Ltd. ethanol and tween 80 was purchased from Merck limited, Mumbai. HPLC acetonitrile was purchased from HiMedia. Triethanolamine, Glycerine and Methylparaben and Propylparaben have purchased from Merck Limited, Mumbai. Micro-organisms (Staphylococcus aureus, E.coli, Proteus vulgaris, Bacillus subtilis) obtained from HiMedia.

\section{Methods}

\section{Preparation of emulsomes}

Emulsomes were prepared by the ethanol injection method. Required quantities of hesperidin, stearic acid, cholesterol and soya lecithin were taken and dissolved in $10 \mathrm{ml}$ of ethanol (Table 1). Take $10 \mathrm{ml}$ of phosphate buffer in another beaker. Now slowly inject this ethanol solution containing hesperidin into buffer solution while stirring. Continue the stirring until the organic phase gets evaporated. Then the solution was subjected to centrifugation. Then collect the sediment and lyophilize the product. Emulsomes were prepared which were spherical in shape.

\section{Preparation of gels}

Emulsomes having high per cent drug release (F5) was formulated as a gel using carbopol 934 and HPMC (Table 2). Carbopol 934, HPMC and water were taken in a beaker and Soaked for $24 \mathrm{hr}$ and stirred using mechanical stirrer at 1000 RPM for $30 \mathrm{~min}$. Then it was neutralised by adding 1-2 drops of triethanolamine. To this gel base glycerine, methyl and propylparaben were added with slow and continuous stirring. ${ }^{16}$

\section{Table 1: Formulation composition of hesperidin loaded emulsomes.}

\begin{tabular}{cccccc}
\hline S.No & $\begin{array}{c}\text { Formulation } \\
\text { code }\end{array}$ & $\begin{array}{c}\text { Stearic } \\
\text { Acid (mg) }\end{array}$ & $\begin{array}{c}\text { Cholesterol } \\
(\mathbf{m g})\end{array}$ & $\begin{array}{c}\text { Soya lecethin } \\
(\mathrm{mg})\end{array}$ & $\begin{array}{c}\text { Drug } \\
(\mathrm{mg})\end{array}$ \\
\hline 1 & F1 & 100 & 50 & 25 & 100 \\
2 & F2 & 100 & 50 & 50 & 100 \\
3 & F3 & 100 & 50 & 75 & 100 \\
4 & F4 & 100 & 50 & 100 & 100 \\
5 & F5 & 100 & 50 & 125 & 100 \\
6 & F6 & 100 & 100 & 100 & 100 \\
7 & F7 & 100 & 125 & 100 & 100 \\
8 & F8 & 100 & 200 & 100 & 100 \\
9 & F9 & 125 & 100 & 100 & 100 \\
10 & F10 & 200 & 100 & 100 & 100 \\
\hline
\end{tabular}

Table 2: Optimization of topical gels using polymers (Carbopol and HPMC).

\begin{tabular}{ccc}
\hline S.No & Carbopol:HPMC & Drug \\
\hline 1 & $1: 1$ & Pure Hesperidin \\
2 & $1: 2$ & Pure Hesperidin \\
3 & $1: 3$ & Pure Hesperidin \\
4 & $1: 4$ & Pure Hesperidin \\
5 & $1: 1$ & Emulsomes of Hesperidin (F5) \\
\hline
\end{tabular}

\section{Evaluation of the emulsomes}

The prepared emulsomes were evaluated for per cent drug content, SEM, FTIR, DSC, Zeta size, zeta potential and in vitro drug release study.

\section{Preparation of stock solution}

$100 \mathrm{mg}$ of hesperidin was dissolved in $100 \mathrm{ml}$ phosphate buffer. Dilutions were made to prepare $1 \mu \mathrm{g}, 2 \mu \mathrm{g}, 3 \mu \mathrm{g}, 4 \mu \mathrm{g}, 5 \mu \mathrm{g}, 6 \mu \mathrm{g}, 7 \mu \mathrm{g}, 8 \mu \mathrm{g}, 9 \mu \mathrm{g}, 10 \mu \mathrm{g}$ by using a buffer. Then the absorbance of these different concentrations of hesperidin was estimated in UV visible spectrophotometer at $285 \mathrm{~nm}$.

\section{Determination of drug content}

$10 \mathrm{mg}$ of emulsomes were taken and add $10 \mathrm{ml}$ of phosphate buffer 7.4. Sonicate the solution for $5 \mathrm{~min}$ and stirred for $30 \mathrm{~min}$. Then the solution was filtered. Dilutions were made and the absorbance was measured at $285 \mathrm{~nm}$ using UV visible spectrophotometer.

\section{SEM analysis}

Morphology of the nanoparticles (emulsomes) was characterized by scanning electron microscopy (SEM). The emulsomes were mounted on aluminium stubs and examined using SEM. ${ }^{17}$

\section{Differential scanning calorimetry}

Small amounts of dried Hesperidin emulsomes powder and pure hesperidin was crimped in a standard aluminium pan and heated from 25 to $400^{\circ} \mathrm{C}$ at a heating rate of $10^{\circ} \mathrm{C} / \mathrm{min}$ under constant heating. ${ }^{18}$

\section{X-Ray diffraction studies}

The crystalline nature of the drug in the pure hesperidin and hesperidin loaded emulsomes was evaluated by using P-XRD studies. The powder $\mathrm{x}$-ray diffractotometer (Regaku-mini plus 500) was done by using $\mathrm{k}$-beta(x2)filtered, cu-k, alpha radiation and a voltage of $30 \mathrm{kv}$ and $15 \mathrm{~mA}^{19}$

\section{Zeta size and Zeta potential}

Nanoparticulate hesperidin emulsomes solution was prepared in double distilled water and sonicated for $30 \mathrm{sec}$ in an ice bath. Size measurements were performed in triplicates. Zeta potential was measured in the same instrument at $25^{\circ} \mathrm{C}$.

\section{In vitro drug release studies}

The in vitro drug release studies were performed using the dialysis membrane method. The dialysis membrane was soaked in water for $24 \mathrm{hr}$ before performing the study. Dialysis membrane was filled with phosphate buffer $\mathrm{pH} 7.4$ and $100 \mathrm{mg}$ of hesperidin emulsomes. Then the dialysis membrane was placed in a beaker containing $\mathrm{pH} 7.4$ phosphate buffer. ${ }^{20}$ The beaker was maintained at $37^{\circ} \mathrm{C}$ temperature and rotated at $100 \mathrm{rpm}$. The samples were collected at time intervals as $0,0.25,0.5$, $0.75,1,2,3,4,5,6,7,8,12,24 \mathrm{hr}$ and an equal amount of the freshly prepared buffer sample was replaced to maintain sink condition and the samples were measured using UV Spectrophotometer at the wavelength of $285 \mathrm{~nm}$.

\section{Evaluation of gels}

The prepared gels were evaluated for drug content, physical evaluation, homogeneity, $\mathrm{pH}$ measurement and ex-vivo permeation studies.

\section{Determination of drug content}

$10 \mathrm{mg}$ of gel loaded with emulsomes of hesperidin was taken to it add 10 $\mathrm{ml}$ of phosphate buffer 7.4 and stirred for $24 \mathrm{hr}$. Then the solution was filtered and diluted with $10 \mathrm{ml}$ of buffer. Absorbance was measured at $285 \mathrm{~nm}$ using UV visible spectrophotometer. ${ }^{21}$ 


\section{Homogeneity}

The emulsomes loaded hesperidin gel was tested for homogeneity by visual inspection if any aggregates in the gel after they have been kept in a container.

\section{$\mathrm{pH}$ of the gel}

The $\mathrm{pH}$ of the emulsomes loaded hesperidin gel was determined by using $\mathrm{pH}$ meter. The $\mathrm{pH}$ of the gel was checked at $1^{\mathrm{st}}, 15^{\text {th }}$ and $30^{\text {th }}$ day after the preparation of gel to find any changes with reference to the time.

\section{Viscosity}

$5 \mathrm{~g}$ of gel were taken in a beaker and the viscosity was checked by using a brook field viscometer by selecting spindle number 64 and the viscosity of gels was noted.

\section{Ex vivo skin permeation studies}

Ex-vivo skin permeation studies were performed to find out the amount of drug permeated through the skin into the body using goat skin which was brought from the local slaughter house and it was kept in buffer $\mathrm{pH} 7.4$ until it is mounted on Franz diffusion cell. Goat skin was taken and hair was removed by using a razor, wiped it with isopropyl alcohol. The skin membrane was placed between the donor and receptor compartment.

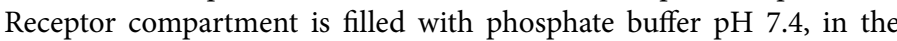
donor compartment, the skin is applied with the emulsomes loaded with hesperidin gel. The diffusion cell was maintained at $37^{\circ} \mathrm{C}$ and the stirring rate was kept at $100 \mathrm{RPM}$ using a magnetic stirrer. $5 \mathrm{ml}$ of samples were taken at regular intervals of time $0,0.25,0.5,1,2,3,4,6,8,12,24 \mathrm{hr}$ and replaced with the same amount of the freshly prepared buffer solution to maintain the sink condition and each sample was studied in triplicate and the average was taken into consideration for further calculation. ${ }^{22}$ The samples were analyzed using UV spectrophotometer at $285 \mathrm{~nm}$. The results were subjected to permeation parameters like permeation coefficient, flux, lag time, diffusion co-efficient. The parameters were calculated by plotting a graph between percentage CDR on the $y$-axis and time on the $\mathrm{x}$-axis. The data obtained in the ex-vivo study was subjected to mathematical models like zero order, First order, Higuchi and Korsmeyer-Peppas model.

\section{In vivo pharmacokinetic study}

The in vivo pharmacokinetic study was done to estimate the concentration of hesperidin in the blood for this the pharmacokinetic parameters were estimated by using RP-HPLC method using water and methanol (50:50) as mobile phase. Blood was withdrawn from the male Wistar albino rats the sample was taken in an Eppendorf tube at different time intervals, sodium citrate was added to prevent coagulation. Then the samples were centrifuged at $15000 \mathrm{RPM}$ for $30 \mathrm{~min}$. The supernatant plasma was collected. ${ }^{23}$ To its internal standard was added. $25 \mu \mathrm{l}$ of the sample was injected and analyzed using RP-HPLC method at $\lambda_{\max } 285 \mathrm{~nm}$. (Group- I pure hesperidin, Group- II emulsomes loaded hesperidin gel).

\section{In vivo anti-inflammatory activity Experimental animals}

Male Wistar albino rats were procured from Sri Venkateswara enterprises Banglore were used in the study. The animals were housed under the standard environmental conditions like ambient temperature $\left(25 \pm 1^{\circ} \mathrm{C}\right)$ and humidity $(55 \pm 5 \%)$ and $12 / 12 \mathrm{~h}$ light-dark cycles. All the experimental procedures and protocols which were used for the anti-inflammatory study was carried out in accordance with the guidelines of CPCSEA and which was approved by the institution of animal ethical committee with Reg. No. 1677/PO/Re/S/2012/CPCSEA/IAEC/25 dt.3.05.2018. The in vivo pharmacodynamic activity was performed to find out the efficacy of emulsomes loaded hesperidin gel in animals. ${ }^{24}$ For the pharmacodynamic study, five groups of male Wistar albino rats were selected each weighing 150-200g. The dose was calculated on the basis of the weight of rats. The inflammation was induced to hind paw by injecting carrageenan using hind paw method. Rats were divided into five groups: Group I- Normal, Group II - Treated as control, Group IIITreated with marketed gel (Marketed Gel), Group IV- Treated with pure hesperidin gel. Group V - Treated with emulsomes loaded hesperidin gel. The paw volume was noted for every one hour using plethysmometer. The inhibition percentage of edema was calculated by using the formula.

$\%$ inhibition of edema $=\frac{\left(\mathrm{V}_{\text {Control }}-\mathrm{V}_{\text {test }}\right)}{\mathrm{V}_{\text {Control }}} \times 100$

\section{RESULTS}

\section{Drug content}

Drug content calculations were done for all the formulations and the highest amount of drug content was found in F5 formulation(98.6\%). The amount of drug content was found to be in the range of $94.5-98.6 \%$.

\section{SEM analysis}

The shape and surface morphology of the prepared emulsomes were analyzed by using scanning electron microscopy. The particles were found to be spherical in shape and the surface shows smooth texture. The SEM images of emulsomes and the surface morphology was shown in Figure 1.

\section{DSC thermogram}

The DSC thermogram of the pure hesperidin drug was obtained at $260^{\circ} \mathrm{C}$ and in the hesperidin emulsomes formulation, it has shown a slight decrease in melting point at $258^{\circ} \mathrm{C}$. This implies that there is no chemical interaction between the drug and the polymer. The DSC thermograms were shown in Figure 2a, $2 b$.

\section{P- XRD study}

$\mathrm{X}$-ray diffractograms of pure hesperidin and hesperidin emulsomes were recorded. This study was performed to find out the crystalanie nature of the drug in pure form and in emulsomes formulation. The diffractograms has shown significant peak at $2 \Theta$ values similar to pure hesperidine. The slight change indicates the reduction of crystal size which implies increase in solubility of drug.

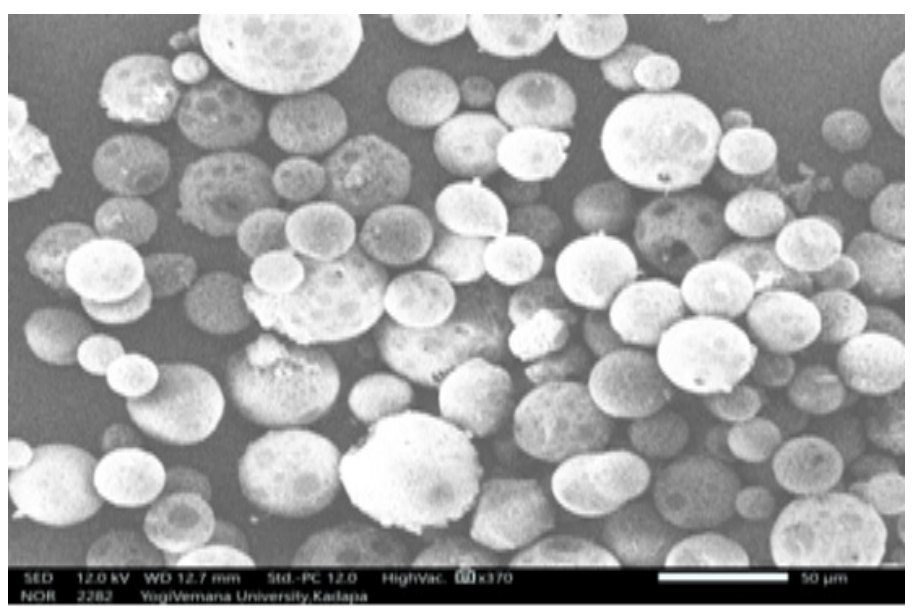

Figure 1: Surface morphology of hesperidin emulsomes, scanning electron microscopy images. 


\section{Zeta size and zeta potential}

The particle size and the zeta potential of the optimized emulsomes formulation was measured using the (Horiba) particle size analyzer, the samples were diluted in triplicates and the size of the optimized F5 emulsomes was found to be $50 \mathrm{~nm}$. The zeta potential of the formulated emulsomes was found to be $-1.8 \mathrm{mV}$ which indicates that the formulation was stable.

\section{In vitro drug release}

All emulsomes formulations were subjected to in vitro drug release studies, the cumulative percentages of drug release were obtained by taking a plot between the time on the $\mathrm{x}$-axis and cumulative percentage of drug release on the $y$-axis. The release studies were performed for all the formulations pure drug, F1, F2, F3, F4, F5 and F6. The amount of drug release was found to be $98.6 \%$ for $6 \mathrm{hr}$ for F5. Cumulative percentage drug release of emulsomes is given in the Figure 3.

\section{Drug content}

The drug content of the prepared gels from X1-X5 was found to be in the range of $92-96 \%$. The drug content of prepared emulsomes is shown in the Table 3.

\section{Homogeneity}

The prepared gels when observed visually they have shown no aggregates and the gel formed was uniform.

$\mathrm{pH}$

The $\mathrm{pH}$ for the formulations of gel X1-X5 showed 6.8 to $7.0 \pm 0.2$ this indicates that the prepared gels were neutral and there is no skin

Table 3: Evaluation of physico-chemical properties of hesperidin loaded emulsomal
gel.
\begin{tabular}{|ccccccc} 
S.No & $\begin{array}{c}\text { Carbopol: } \\
\text { HPMC }\end{array}$ & Drug & $\begin{array}{c}\text { Viscosity } \\
\text { (cp) }\end{array}$ & Appearance & $\begin{array}{c}\text { Drug } \\
\text { Content }\end{array}$ & $\mathrm{pH}$ \\
\hline 1 & $\mathrm{X}_{1}$ & Pure Hesperidin & 8,640 & Homogenous & $93.5 \pm 0.69$ & 7.0 \\
2 & $\mathrm{X}_{2}$ & Pure Hesperidin & 8,820 & Homogenous & $92.8 \pm 1.38$ & 7.0 \\
3 & $\mathrm{X}_{3}$ & Pure Hesperidin & 9,600 & Homogenous & $94.9 \pm 0.87$ & 7.0 \\
4 & $\mathrm{X}_{4}$ & Pure Hesperidin & 27,960 & Homogenous & $96.0 \pm 3.54$ & 6.8 \\
5 & $\mathrm{X}_{5}$ & $\begin{array}{c}\text { Hespiridin loaded } \\
\text { Emulsomes(F5) }\end{array}$ & 8,745 & Homogenous & $95.5 \pm 0.53$ & 7.0 \\
& & & & & \\
\hline
\end{tabular}

The values are given as mean $\pm \mathrm{SD}, n=3$

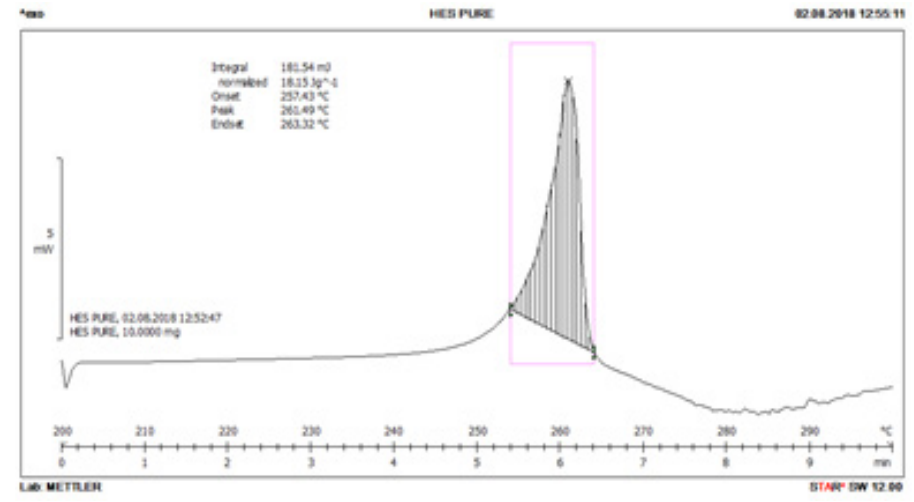

Figure 2a: DSC of pure hesperidin.

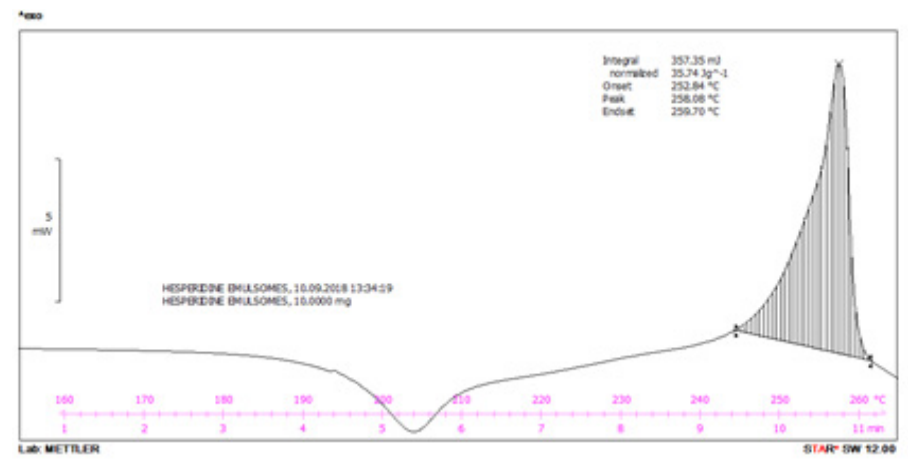

Figure $\mathbf{2 b}$ : DSC of hesperidin emulsomes.

Figure 2a,2b: DSC Differential scanning calorinetry themograms.
Table 4: Percent edema inhibition of hesperidin marketed gel, hesperidin emulsomes gel and hesperidin gel.

\begin{tabular}{cccc}
\hline $\begin{array}{c}\text { Time } \\
\text { (hr) }\end{array}$ & Marketd gel & $\begin{array}{c}\text { Hesperidin- } \\
\text { Emulsomal gel }\end{array}$ & Hesperidin-Gel \\
\hline 0 & 0 & 0 & 0 \\
1 & 36.9 & 43.91 & 10.69 \\
2 & 51.9 & 71.62 & 25.64 \\
3 & 77.3 & 91.3 & 40 \\
\hline
\end{tabular}

values were expressed as mean \pm SEM. $P<0.05$ compared with disease control, ANOVA $\left(\mathrm{F}_{\mathrm{c}}>\mathrm{F}_{\mathrm{t}}\right)$ there is a significant difference.

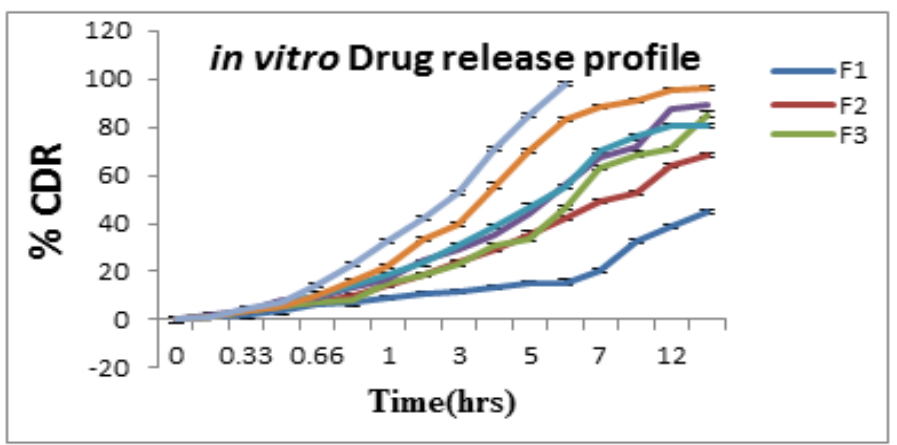

Figure 3: Comparative in vitro dissolution profile of different hesperidin loaded emulsomes formulations, mean $\pm S D, n=3$. 
irritation when applied to the skin. The $\mathrm{pH}$ of prepared emulsomes is shown in the Table 3.

\section{Viscosity}

The viscosity of gel formulations was measured by using a Brookfield viscometer and the viscosity of the prepared gels was found to be in the range 8640 to $27960 \mathrm{cps}$ as the concentration of the carbopol polymer increases the viscosity of the gel increases gradually. The viscosity of prepared emulsomes gel using different concentrations of carbopol and HPMC is shown in the Table 3.

\section{Ex vivo skin permeation studies}

The hesperidin loaded emulsomes gel and pure hesperidin gel were subjected to ex-vivo skin permeation studies using goatskin. The cumulative per cent of drug permeated through the skin for gel loaded with F5 formulation was found to be $98.9 \%$ for four hours and for the pure hesperidin gel it showed $45 \%$ for $24 \mathrm{hr}$. The permeation parameters were calculated from a graph by plotting the cumulative per cent drug release on the $\mathrm{y}$-axis versus time on the $\mathrm{X}$-axis. From the slope of the graph flux value was found to be $12.3 \mu \mathrm{g} / \mathrm{cm}^{2} / \mathrm{h}$ for hesperidin emulsomal gel and for pure hesperidin gel it was found to be $5.53 \mu \mathrm{g} / \mathrm{cm}^{2} / \mathrm{h}$ the flux value of hesperidin emulsomal gel was more when compared to the pure hesperidin gel this indicates more permeation of drug. The permeation coefficient, lag time and diffusion coefficient was calculated for hesperidin emulsomal gel and pure hesperidin gel and the values are as follows $(4.24,1.25 \mathrm{~kg})(0.02 \mathrm{~h}, 0.86 \mathrm{~h})\left(0.032,0.16 \mathrm{~mol} / \mathrm{m}^{3}\right)$. It was found that emulsomes loaded hesperidin gel release follows zero order mechanism which governs the release of the drug. From the equation $R^{2}=0.99$ the drug release follows Higuchi model which indicates it follows the diffusion mechanism (Fickian $n<0.5$ ). The hesperidin loaded emulsomal gel showed the best release when compared to pure hesperidin gel.

\section{Pharmacodynamic studies}

The pharmacodynamic activity of a pure hesperidin gel, hesperidin loaded emulsomes and marketed emulgel (Voveran) was carried out using male Wister albino rats. From the in vivo pharmacodynamic study, the percentage inhibition of edema in group I rats are $0 \%$ as it is not treated. The percentage inhibition for group III shows inhibition of $40 \%$. Whereas group IV and V rats are more inhibition of $77.3 \%, 91.3 \%$ because it was treated with marketed gel and prepared gel. The percentage inhibition of group V rats is more compared to group III because as the solubility increases, permeation also increases thus it shows effective inhibition of loaded gel. The data given in Table 4 were were subjected to ANOVA, it was found that the F ratio was 1.09 ( $\mathrm{fc}<\mathrm{ft}$ ). So there is a significant difference between the pure hesperidin and hesperidin loaded emulsomal gel. The percentage of inhibition of hesperidine emulsomal gel was found to be two folds more when compared to the plain hesperidin gel.

\section{Pharmacokinetic activity}

The pharmacokinetic parameters for gel containing pure hesperidin and gels containing emulsomes of hesperidin were calculated using plasma concentrations. It was found that pure hesperidin gel has $\mathrm{C}_{\max }, \mathrm{T}_{\max }, \mathrm{t}_{1 / 2}$, AUC as follows $9 \mathrm{mcg} / \mathrm{ml}, 4 \mathrm{hr}$ and $5.2 \mathrm{hr}, 85 \mathrm{mcg} . \mathrm{hr} / \mathrm{ml}$. Gel containing emulsomes of hesperidin has $\mathrm{C}_{\max }, \mathrm{T}_{\max }, \mathrm{t}_{1 / 2}$, AUC of pure drug was found to be $14 \mathrm{mcg} / \mathrm{ml}, 2 \mathrm{hrs}$ and 5.6hr, $106 \mathrm{mcg} . \mathrm{hr} / \mathrm{ml}$. HPLC chromatogram of hesperidin in rat plasma after topical application of gel loaded with hesperidin emulsomes is shown in Figure 4.

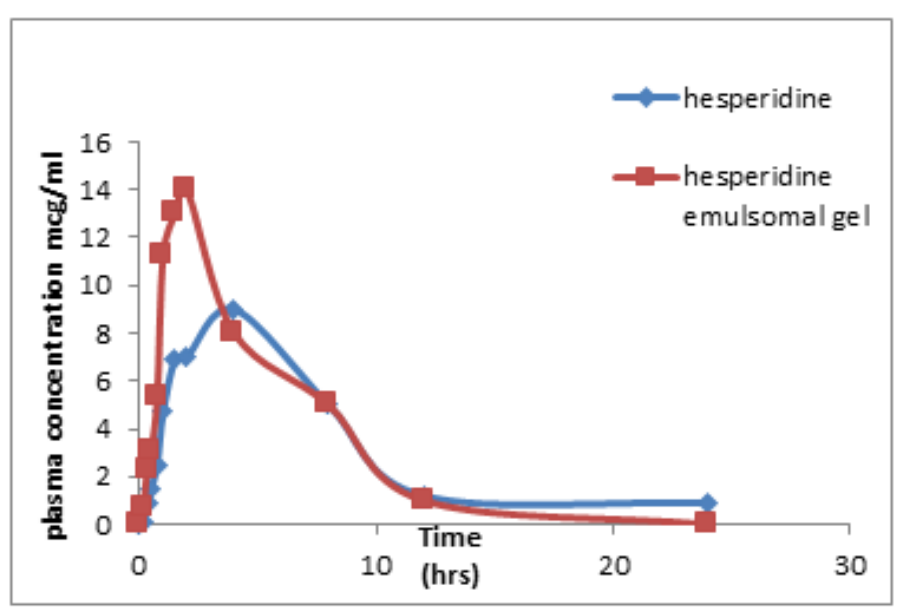

Figure 4: Plasma concentration time profile of pure hesperidin and hesperidin loaded emulsomal gel in rats $n=6$, Data presented as mean \pm SD.

\section{DISCUSSION}

The hesperidin loaded emulsomes were prepared using ethanol injection method using stearic acid, cholesterol, soya lecithin and hesperidin. The formed emulsomes were found to be spherical in shape, with smooth texture with a size of $50 \mathrm{~nm}$. The effect of the drug to polymer ratio on the particle size of emulsomes was evaluated as the concentration of the polymer increases the size of the emulsomes increase. Zeta potential of the optimized emulsomes $\mathrm{F} 5$ was found to be $-1.8 \mathrm{mV}$ which indicates that the particles are stable. DSC thermograms of pure hesperidin and hesperidin emulsomes has shown same melting point which indicates that there is no incompatibility between drug and polymer. To confirm the crystalline state of hesperidin and hesperidin emulsomes powder XRD was performed with pure hesperidin and hesperidin loaded emulsomes. The powder XRD pattern of pure hesperidin showed sharp and significant, intense peaks at $2 \Theta$ values of $10,16,21,29$ which are the characteristic nature of pure hesperidin. The above mentioned $2 \Theta$ values indicates that there is no significant difference between the pure hesperidin and hesperidin emulsomes. The only slight difference in the intensity peaks in diffractograms of particles between pure hesperidin and hesperidin emulsomes was because due to a reduction in the size of crystals, this reduction in size indicates the improvement in dissolution rate and its bioavailability. The in vitro drug release studies reveal that the amount of drug release was found to be $98.6 \%$ of $\mathrm{F} 5$ formulation and for F3 89.3\%, F2 85.3\%, F1 68.2\%, F4 80.64\%, F5 98.6\% and pure drug 45\% at the end of $24 \mathrm{hr}$. The optimized F5 formulation is converted into the gel using the polymers carbopol and HPMC. As the concentration of the carbopol increases the viscosity increases and after certain concentration, the viscosity becomes more and aggregates are formed which was in the range of 8,640-27,960. The polymer carbopol was selected because of its good bioadhesive property as it remains on the skin for longer periods of time and drug leakage was also minimum due to carbopol 943 gel high viscosity. The percentage drug diffusion of hesperidin emulsomes loaded gel was found to be $98 \%$ as the solubility increases, it permits the drug easily through the skin and thus the amount of drug release was more compared to pure hesperidin gel and a good linearity of 0.844 was observed with zero order it follows zero order release and the slope of the Higuchi model shows that it follows the diffusion pattern of drug release and the value indicates that it follows the Fickian diffusion mechanism. The outermost layer and the primary layer of the skin is stratum corneum through which most of the drugs penetrate into the body. This starum corneum is compositionally biomembrane which consists layers of corneocytes and anchored in lipid matrix. This composition makes 
the skin less permeable to the water and other materials when compared to the other layers. As hesperidin belongs to BCS class II drug, it has less solubility and highly permeation capacity the drug permeation through stratum corneum of the skin can be done by two mechanisms one is by augmentation of skin permeability and secondly by activation of concentration independent transport driving system, normally some penetration enhancers are used to enhance the permeation of drug through the skin. ${ }^{25}$ Which causes some skin irritation instead of this we developed a vesicular drug delivery system with high permeation capacity. From pharmacokinetic parameters, the in vivo bioavailability of hesperidin nanogel was found that $\mathrm{C}_{\max }, \mathrm{T}_{\max }, \mathrm{t}_{1 / 2}$, AUC $14 \mathrm{mcg} / \mathrm{ml}$, $2 \mathrm{hr}, 5.6 \mathrm{hr}$ and AUC as $106 \mathrm{mcg} / \mathrm{ml}$. As the emulsomes have more permeation the amount of the drug enters into blood circulation was more compared to pure hesperidin gel. The data ofpharmacodynamic study given were subjected to ANOVA, it was found that the F ratio was $1.09(\mathrm{fc}<\mathrm{ft})$. So there is a significant difference between the pure hesperidin and hesperidin loaded emulsomes gel.

\section{CONCLUSION}

The novel promising hesperidin nanoemulgel showed as a better carrier for topical drug delivery system. The optimized formulation has shown better stability through out the study and it can also act as good alternative for the oral NASAID drug delivery system which has side effects like cardiac arrest, gastric irritation, renal problem and also bypasses the first pass metabolisam. Hespiridine loaded emulsomes prepared with phospholipid, cholesterol and sstearic acid by ethanol injection method were successfully incorporated into the topical gel. The hesperidin emulgel character showed therapeutically effective treatment for anti-inflammatory activity when compared to plain hesperidin gel and marketed voveran Emulgel.

\section{ACKNOWLEDGEMENT}

Authors expresses their acknowledgements to Institute of Pharmaceutical Technology, DST CURIE center, Sri Padmavathi MahilaVisvaVidyalayam (Women's University) for providing necessary facilities to carryout this research work.

\section{CONFLICT OF INTEREST}

The authors declare that there are no conflict of interest. This research has not received any specific grant from any agency in public, commercial, not for profit sectors.

\section{ABBREVIATIONS}

DSC: Differential scanning calorimetry; HPMC: Hydroxy propyl methyl cellulose; P-XRD: Powder X-Ray Diffractometer; AUC: Area under curve; NSAID: Non-steroidal anti-inflammatory drug; FT-IR: Fouriertransform infrared spectroscopy.

\section{REFERENCES}

1. Olszanecki R, Gebska A, Kozlovski VI, Gryglewski RJ. Flavonoid and nitric oxide synthetase. Physiol Pharmacol. 2019;53(1):571-84.

2. Chen MC, Ye YI, Guang JI, Jian-Wen LIU. Hesperidin upregulates heme oxygenase- 1 to attenuate hydrogen peroxide-induced cell damage in hepatic L02 cells. J Agric Food Chem. 2010;58(6):3330-5.

3. Ferrero-Miliani L, Nielsen $\mathrm{OH}$, Andersen PS, Girardin SE. Chronic inflammation: Importance of NOD2 and NALP3 in interleukin-1beta generation. Clin Exp Immunol. 2007;147(2):227-35

4. Burke A, Smyth E, Fitzgerald GA. Pharmacological basis of Therapeutics (11th edn), McGraw-Hill. New York. 2006;671-716.

5. Heiati HP, Tawashi R. Evidence for phospho- lipid bilayer formation in solid lipid nanoparticles formulated with phospholipid and triglyceride. Pharm Res. 1996;13(9):1406-10.

6. Mullen AB, Carter KC, Baillie AJ. Comparison of the efficacies of various formulations of amphotericin $B$ against murine visceral leishmaniasis. Antimicrob Agents Chemother. 1997;41(10):2089-92.

7. Amselem S, Yogev A, Zawoznik E, Friedman D. Emulsomes, a novel drug delivery technology. Proceedings of the International Symposium on Controlled Release of Bioactive Materials. 1994;21:1368-9.

8. Flückiger FA, Hanbury D. Pharmacographia: A history of the Principal Drugs of Vegetable Origin. Macmillan and Co. London, UK. 1996.

9. Barthe GA, Jourdan PS, Mclntosh CA, Mansell RL. Radioimmunoassay for the quantitative determination of hesperidin and analysis of its distribution in Citrus sinensis. Phytochemistry. 1988;27(1):249-54.

10. Bhalla NP, Dakwake RN. Chemotaxonomy of Indigofera Linn. J Indian Bot Soc. 1978;57:180-5.

11. Pawlowska L. Flavonoids in the leaves of polish species of the genus Betula L. III. The Flavonoids of B. oycoviensis Bess. leaves. Acta Soc Bot Pol. 1980;49(3):311-20

12. Kokkalou E, Kapetanidis I. Flavonoids of the arial parts of Acinos suaveolens Pharm Acta Helv. 1988;636:170-3.

13. Kawaguchi K, Kikuchi S, Takayanagi K, Yoshikawa T, Kumazawa Y. Colony stimulating factor inducing activity of hesperidin. Planta Med. 1999;65(04):3656.

14. Boisseau MR. Recent finding in the pathogenesis of venous wall degradation Int Angiol. 2002;21:33-9.

15. Cao M, Ren L, Chen G. Formulation Optimization and ex vivo and in vivo Evaluation of Celecoxib Microemulsion-Based Gel for Transdermal Delivery. AAPS Pharm Sci Tech. 2017;18(6):60-71.

16. Sarah SY, Indira MY. Formulation and optimization of quercetin loaded emulsomes topical gel: EX vivo. Pharmacodynamic and pharmacokinetic studies. 2019;V11(5):156-65.

17. Blanco-García E, Otero-Espinar FJ, Blanco-Méndez J, Leiro-Vidal JM, LuzardoÁlvarez A. Development and characterization of anti-inflammatory activity of curcumin-loaded biodegradable microspheres with potential use in intestinal inflammatory disorders. Int J of Pharmaceutics. 2017;518(1-2):86-100.

18. Yinghui L, Changshan S, Yanru H, Gongying J, Li Z, Siling W. Mechanism of Dissolution Enhancement and bioavailability of Poorly water soluble celecoxib by preparing stable amorphous nanoparticles. J Pharm Pharmaceut Sci. 2010;13(4):589-606.

19. Girish K, Asish D. Design, Development and evaluation of ion-activated in situ gel of moxifloxacin hydrochloride. Asi J of Pharma and Clin Research. 2019:3:94-103.

20. Liu Y, Sun C, Hao Y, Jiang T, Zheng L, Wang S. Mechanism of dissolution enhancement and bioavailability of poorly water-soluble celecoxib by preparing stable amorphous nanoparticles. J Pharm and Pharmaceu Science. 2010;13(4):589-606

21. Jaya S, Divas S. Formulation and in vitro evaluation of matrix tablets of metoclopramide hydrochloride. Int J Applied Pharm. 2018;11(2):25-30.

22. Tuncay, Calis S, Kas M. In vitro and in vivo evaluation of diclofenac sodium loaded albumin microspheres. J Microencapsulation. 2000;17(2):145-55

23. Khalid A, Pradeep R, Franscisco T, Roberta C. Cyclodextrin based emulsomes for delivery of resebertol: In vitro characterization, stability, cytotoxicityand permeation study. AAPS Pharma Sci Tech. 2011;12:279-86.

24. Koçkaya EA, Selmanoğlu G, Kısmet K, Akay MT. Pathological and biochemical effects of therapeutic and supratherapeutic doses of celecoxib in Wistar albino male rats. Drug Chem Toxicol. 2010;33(4):410-14.

25. Ivanova NA, Hyusein Y, Slavov IZ, Kumanov I. Biopharmaceutical and marketing evaluation of diosmin-and hesperidin-containing products on the Bulgarian market. Scripta Scientifica Pharmaceutica. 2019;5(2):61-70.

Article History: Submission Date : 05-08-2020; Revised Date : 11-09-2020; Acceptance Date : 03-10-2020.

Cite this article: Sujitha YS, Muzib YI. Preparation of Topical Nano Gel Loaded With Hesperidin Emulsomes: In vitro and in vivo Studies Int. J. Pharm. Investigation, 2020;10(4):500-5. 